\title{
Synthesis of Chiral Gold Nanoparticle by Direct Reduction with L and D-Serine and Enhanced Anti-Mycobacterial Activity by D-Serine Protected Gold Nanoparticle
}

\section{Koushik Mukherjee and Alok Kumar Sil*}

Department of Microbiology, University of Calcutta, Kolkata, India

\begin{abstract}
Current situation demands more biocompatible and non-toxic nanostructures. Towards this the present study describes the synthesis of gold nanoparticle by direct reduction of tetrachloroauric acid with both D- and L-enantiomeric forms of the amino acid serine. The formation of nanoparticle was confirmed by examining different physical characteristics like surface plasmon resonance. Moreover, the retention of chirality of the reduced particle was also evident from circular dichroism spectroscopy experiment. In addition, the shielding of the nanoparticle by respective amino acid was confirmed by Scanning Electron Microscope and Energy Dispersive X-ray Analysis (SEM-EDAX). As D-serine is known to be active against Mycobacteria, examination of the biological activity of the D-serine protected particle was performed against the same. The result showed higher inhibitory activity of the particle against Mycobacterium smegmatis than D-serine alone. Thus the present study describes a new protocol for the synthesis of chiral gold nanoparticle that can be beneficial in enantioselective biological applications.
\end{abstract}

Keywords: Chiral nanoparticle, Biocompatible gold nanoparticle; Mycobacteria; D-serine; L-serine

\section{Abbreviations}

CD: Circular Dichroism; DLS: Dynamic Light Scattering; EDAX: Energy Dispersive X-ray Analysis; GNP: Gold Nanoparticle; Mycobacterium smegmatis: M. smegmatis; SEM: Scanning Electron Microscope; SPR: Surface Plasmon Resonance; TEM: Transmission Electron Microscope

\section{Introduction}

The use of nanomaterial in biology is an emerging field with potentially wide range of applications, such as bioimaging, biosensing, gene delivery and in medicine [1,2]. In particular, use of gold nanoparticle (GNP) as an almost inert and biocompatible vehicle in the field of drug delivery and therapeutic purpose is of much interest to clinical researchers [1]. Increased use of synthetic nanomaterials in biological application demands a more biocompatible, safe and effective nanostructure with less hazardous byproducts of synthesis reactions. In this context, besides classical methods of synthesis $[3,4]$, methods of GNP synthesis using biocompatible agents as reducing as well as protection agents are gaining importance. Moreover, biological functions of GNP depend highly on its size, shape and stability. Thus control of size and morphology of GNPs is of prime importance for optimizing its applications in biological fields. These properties of nanoparticle are completely dependent on the type and concentration of reducing agent used in the reaction. Therefore, exploring new protocols for the synthesis of GNPs by different non-toxic biomaterials like amino acids, primary amines, and biopolymers are becoming useful and popular [5-7]. Several reports are already available showing amino acid assisted synthetic routes of GNP formation. Xu et al. reported synthesis of nanowire using glutamic acid and histidine as capping agents [8]. The synthesis of water dispersed GNP by direct reduction with glutamic acid and its bioconjugation ability was reported by Wangoo et al. [9]. Also, there are reports of synthesis of GNP by direct reduction with amino acids or capping of chemically synthesized GNP with the amino acids like tryptophan, lysine, aspartic acid, cysteine etc [10-12].

Besides synthesis of biocompatible nanoparticle, the use of nanoparticle as drug delivery vehicle has emerged with great importance. GNP-mediated enhancement of the anti-microbial activity of antibiotics has been documented [13]. Existing literature also documents direct and efficient delivery of different nanoparticle coated with anti-tubercular drugs [14-16]. In majority of these cases, the nanoparticle served as an efficient delivery vehicle. In some cases nanoparticle enhances the anti-microbial potential by allowing the release of drug for sustained period. In many cases nanoparticles increase the membrane permeability of a particular drug [17], and thus the nano conjugated drug may even become effective against drugresistant strains. Consistent with this hypothesis, several reports of successful inhibition of drug-resistant strains by metal nanoparticles and its drug conjugate have already been reported $[18,19]$.

Since chirality is an important factor in molecular recognition, it has key applications in the fields of chemistry, biology and medicine [20]. Thus there is an increasing need for synthesizing chiral metal nanoparticles. Chiral nanoparticles and nano structures are being used in asymmetric catalysis, chiral separation, enantioselective detection, optoelectronics and development of biosensor as well as enantioselective delivery of drugs [2,21-25]. Different routes of synthesis have been followed in synthesizing metal or polymeric nanoparticle with chiral surface. In a previous work, preparation of polymeric chiral nanoparticles of amino acids derivatives, which was not adsorbed on any metal surface, was described and its enantioselective binding property was assessed [26]. There is report showing the synthesis of silver nanoparticle capped with L-cysteine that was used for enantiospecific recognition of D and L-histidine [27]. Besides, the transfer of chirality of gold nanocluster to a non-chiral adsorbate has also been documented

*Corresponding author: Alok Kumar Sil, Department of Microbiology, University of Calcutta, 35, B. C. Road, Kolkata-700 019, India, Tel: +91-983-183-6050; E-mail: alokksil7@gmail.com

Received August 06, 2015; Accepted September 16, 2015; Published September 21, 2015

Citation: Mukherjee K, Sil AK (2015) Synthesis of Chiral Gold Nanoparticle by Direct Reduction with L and D-Serine and Enhanced Anti-Mycobacterial Activity by D-Serine Protected Gold Nanoparticle. Mod Chem appl 3: 165. doi:10.4172/23296798.1000165

Copyright: (c) 2015 Mukherjee $\mathrm{K}$, et al. This is an open-access article distributed under the terms of the Creative Commons Attribution License, which permits unrestricted use, distribution, and reproduction in any medium, provided the original author and source are credited. 
[28]. In a separate report Kumar et al. showed chiral imprinting of gallium nanoparticle with D and L-tryptophan [29]. In this study for the first time we describe the synthesis and stabilization of GNP by reduction with both the $\mathrm{D}$ - and $\mathrm{L}$-enantiomeric forms of serine and also report the retention of chirality in GNP-conjugated with these amino acids. D-serine has long been reported to have an inhibitory activity against different species of Mycobacteria and some other bacteria from different genus. It is reported that $\mathrm{D}$-serine inhibits the racemization of alanine by inhibiting alanine racemase, an enzyme essential for the synthesis of uridine diphosphate- $\mathrm{N}$-acetylmuramylpentapeptide, a precursor of cell wall mucopeptide and thus inhibit cell wall synthesis in several bacteria [30]. The current study also reports an enhancement of anti-mycobacterial activity of D-serine when delivered as GNPcoated with this amino acid.

\section{Materials and Methods}

\section{Reagents}

Gold (III) chloride trihydrate $\left(\mathrm{HAuCl}_{4} \cdot 3 \mathrm{H}_{2} \mathrm{O}\right)$ was purchased from Sigma-Aldrich. L-serine and D-serine was purchased from Sisco Research Laboratory, India. MilliQ water used in this study was obtained from Milli-Q ultra pure system (Millipore, India).

\section{Synthesis of gold nanoparticle (GNP)}

An aqueous solution of GNPs was prepared by reducing tetrachloroauric acid $\left(\mathrm{HAuCl}_{4}\right)$ with $\mathrm{L}$-and $\mathrm{D}$-serine under refluxing to yield colloidal gold particles. For this, $20 \mathrm{ml}$ of $1 \mathrm{mM}$ tetrachloroauric acid in Milli-Q water was taken in a reaction flask and refluxed for 15 min with $20 \mathrm{ml}$ aqueous solution of amino acid of different molarities (a: $25 \mathrm{mM}$, b: $50 \mathrm{mM}$, c: $100 \mathrm{mM}$ ). The reduction of the gold metal ions $\left(\mathrm{Au}^{3+}\right)$ to yield GNPs $\left(\mathrm{Au}^{0}\right)$ was confirmed by the appearance of a dark cherry-red to blue color in the different colloidal solutions. Colloidal gold solution was subjected to centrifugation and the conjugate was washed with deionized water to remove any uncoordinated amino acid. The conjugate was then dried and subjected to characterization using various physico-chemical techniques.

\section{Characterization of GNPs}

The UV-Vis absorbance spectra of aqueous GNPs were recorded using a Jasco V-530 UV/VIS spectrophotometer. Particle size distribution was analyzed using particle size analyzer (Malvern Zetasizer) by illuminating the colloidal gold solution with He-Ne laser $(633 \mathrm{~nm})$ in a sample cell. The corresponding zeta potential of the GNP was measured in a Beckman-Coulter Delsa" Nano particle size and zeta analyzer. Transmission electron microscopic studies were carried out using Philips Fei Technai Spirit Transmission Electron Microscope (TEM) operating at $80 \mathrm{kV}$ accelerating voltage. Samples were prepared by placing a drop of GNPs solutions on carbon-coated TEM grids. The films on the TEM grids were allowed to dry overnight at room temperature before analysis.

\section{Circular Dichroism (CD)}

CD spectroscopy was performed in a Jasco CD spectrometer (J815 ) at a temperature of $20^{\circ} \mathrm{C}$ in cell of length $10 \mathrm{~mm}$. Briefly, GNP reduced by $100 \mathrm{mM}$ of both $\mathrm{L}$ and $\mathrm{D}$-serine was completely sedimented by centrifugation and washed twice by Milli-Q water. Thereafter, the pellet was dissolved in an appropriate volume of Milli-Q water. Aliquots from both preparations were subjected to $\mathrm{CD}$ analysis. For the $\mathrm{CD}$ analysis of pure $\mathrm{L}$ and $\mathrm{D}$-serine, $10 \mathrm{mM}$ concentration of each amino acid was used.

\section{Scanning Electron Microscope and Energy Dispersive X-ray (SEM-EDAX) analysis}

Amino acid encapsulated GNP was dropped onto adhesive carbon tape and vacuum-dried overnight. The surface was evaluated by SEMEDS (JSM-7600F, JEOL Ltd., Japan) operated at $5 \mathrm{kV}$.

\section{Estimation of unreacted amino acid in the reaction mixture}

After eliminating GNP by centrifugation at $15,000 \mathrm{rpm}$ for $15 \mathrm{~min}$, the amount of unreacted amino acid was estimated by a ninhydrinbased assay. Briefly, $200 \mu \mathrm{l}$ of ninhydrin solution $(0.35 \%$ in ethanol) was added to $1 \mathrm{ml}$ of amino acid sample and heated at $80-100^{\circ} \mathrm{C}$ for $4-7$ min with mild stirring. Thereafter, the solution was cooled in a water bath and the absorbance was measured at $570 \mathrm{~nm}$. A standard curve for amino acid concentration versus absorbance was generated by reacting known concentrations of amino acid with ninhydrin $(6 \mathrm{mM}, 12.5 \mathrm{mM}$, $25 \mathrm{mM}$ and $100 \mathrm{mM}$ ). The standard curve was used to calculate the concentration of amino acid in the supernatant.

\section{Flocculation assay}

To determine the critical flocculation concentration of $\mathrm{D}$ - serine protected GNPs, $60 \mu \mathrm{l}$ of $\mathrm{NaCl}$ of different strengths $(0-3 \mathrm{M})$ was added to $600 \mu \mathrm{l}$ of amino acid protected GNPs and was incubated for $1 \mathrm{hr}$ at room temperature under mild shaking. Aggregation was assessed by monitoring changes in the characteristic GNP plasmon frequency. The threshold $\mathrm{NaCl}$ concentration in the colloidal gold that caused the aggregation of the particles was determined as the critical flocculation concentration.

\section{Assay of anti-mycobacterial activity of D-serine protected GNP}

Middlebrooks 7H9 base broth [ammonium sulphate $(0.5 \mathrm{~g} / \mathrm{L})$, di-sodium hydrogen phosphate $(1.5 \mathrm{~g} / \mathrm{L})$, potassium di-hydrogen phosphate $(1.5 \mathrm{~g} / \mathrm{L})$, tri-sodium citrate $(0.4 \mathrm{~g} / \mathrm{L})$, sodium glutamate $(0.5 \mathrm{~g} / \mathrm{L})$, calcium chloride $(0.011 \mathrm{~g} / \mathrm{L})$, sodium hydroxide $(0.0002$ $\mathrm{g} / \mathrm{L})$, magnesium sulphate $(0.005 \mathrm{~g} / \mathrm{L})$, zinc sulphate $(0.001 \mathrm{~g} / \mathrm{L})$, copper sulphate $(0.001 \mathrm{~g} / \mathrm{L}$ ] supplemented with glycerol $(0.5 \%)$ and Bovine Serum Albumin (BSA) (0.025\%) was used for the cultivation of Mycobacterium smegmatis (M. smegmatis) $\mathrm{mc}^{2} 155$.

For anti-mycobacterial assay of D-serine alone and D-serine protected GNP, $1 \mathrm{ml}$ Middlebrook 7H9 medium was supplemented with either varying concentrations of D-serine $(6 \mathrm{mM}, 12 \mathrm{mM}, 24 \mathrm{mM}$, $48 \mathrm{mM})$ or GNP containing varying amounts of $\mathrm{D}$-serine $(0.5 \mathrm{mM}, 1.0$ $\mathrm{mM}, 2.0 \mathrm{mM}, 4.0 \mathrm{mM}$ ) (as calculated by ninhydrin experiment) and the growth of $M$. smegmatis was examined after $48 \mathrm{hr}$ of incubation at $37^{\circ} \mathrm{C}$.

\section{Results}

\section{Synthesis and characterization of GNP by direct reduction with $D$ - and L-serine}

An aqueous solution of colloidal gold was prepared by reducing tetrachloroauric acid with both $\mathrm{D}$ - and L-serine as described in Materials and Methods. As expected GNP generated by different concentration of amino acid varied in size which is visually evident from difference in color of the nanoparticle solution (Figures 1a and 2a). Particles synthesized with a lower concentration of reducing agent (25 mM) yielded larger nanoparticles resulting in bluish-purple color solution, whereas, particle synthesized using higher concentration of reducing agent $(100 \mathrm{mM})$ yielded smaller particles which resulted in characteristic red color solution. This is also supported by the red shift 
of absorption maxima due to Surface Plasmon Resonance (SPR) of GNP [31] (Figures 1a and 2a). Figures 1a and 2a show the UV-Vis spectra recorded from the colloidal gold solutions reduced with different concentrations of $\mathrm{D}$ - and L-serine, respectively. A strong absorption at nearly $540 \mathrm{~nm}$ is observed for all spectra. As expected, a red shift for particles synthesized with lower concentration of amino acid was clearly observed $[9,31]$. The hydrodynamic radius of the GNPs were recorded by Dynamic Light Scattering (DLS) and the average radius for D-serine reduced particle was found to be $98.3,85.4$ and $57.9 \mathrm{~nm}$ for $25 \mathrm{mM}, 50 \mathrm{mM}$ and $100 \mathrm{mM}$ amino acid, respectively (Figure 1b). The same was found to be $80.23,79.24$ and $42.12 \mathrm{~nm}$, respectively for L-serine (Figure 2b). Particle synthesized by $100 \mathrm{mM}$ of both D-serine and L-serine was visualized under TEM and the average size was found to be $52.8 \pm 5.33 \mathrm{~nm}$ (Figure 1c) and $29.6 \pm 4.78 \mathrm{~nm}$, respectively (Figure 2c). The shape of a single particle was found to be more like hexagonal than a perfect sphere (Figures $1 c$ and $2 c$ ).

The GNPs so produced may retain the chirality of its reducing agent. To test this, GNPs synthesized by reduction with amino acids were subjected to $\mathrm{CD}$ analysis. The CD spectrum of pure $\mathrm{D}$ - and L-amino acid clearly showed a peak at $203 \mathrm{~nm}$ with an ellipticity of opposing magnitude (Figure 3). Similarly, GNP synthesized by amino acids also showed $\mathrm{CD}$ spectrum symmetric to $\mathrm{X}$-axis with a positive peak for L-serine and negative peak for D-serine (Figure 3). This result indicated the retention of chirality of these two amino acids even they are conjugated with GNP. Thus our method of GNP preparation preserves chirality of the amino acids. Therefore, the current study not only provides a non-hazardous method for synthesizing GNP, but also provides a simple method for the synthesis of chiral nanoparticle. In the subsequent experiments we focused only on GNP synthesized using D-serine.

\section{Shielding of the GNP by D-serine was efficient and stable}

Generally, the reducing agent used in nanoparticle synthesis also participates in shielding and protecting the nanoparticle in solution [9]. Consistent with this, in the previous section we observed retention of the chirality by the GNPs. It indicated a possible protection of GNP by its respective reducing agent, i.e., $\mathrm{L}$ or $\mathrm{D}$-amino acids. To

(a)

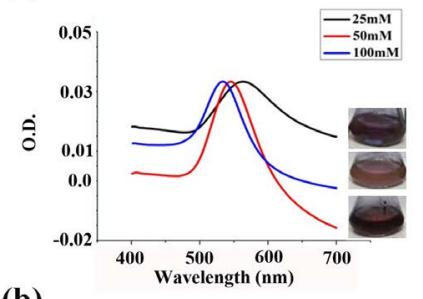

(c)

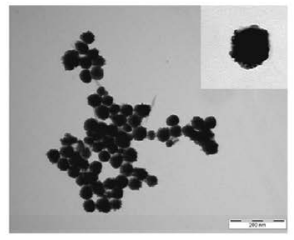

(b)

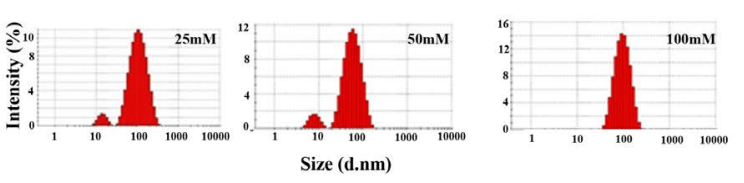

Figure 1: Physical characterization of GNPs synthesized using different concentrations of D-serine. (a) Colour and SPR of GNPs. Different concentrations of D-serine are indicated with different coloured lines: blue line indicates $100 \mathrm{mM}$, red line indicates $50 \mathrm{mM}$ and black line indicates $25 \mathrm{mM}$. (b) Average hydrodynamic radius of GNPs. Average hydrodynamic radiuses of GNPs were determined by DLS (left panel is for $25 \mathrm{mM}$, middle panel is for $50 \mathrm{mM}$, and right panel is for $100 \mathrm{mM}$ ). (c) Transmission electron microscopic image of GNP synthesized using $100 \mathrm{mM}$ D-serine. Shape of a single particle is showed in inset. confirm the protection of GNP by amino acid, we performed SEMEDAX experiment for the elemental analysis of nanoparticle surface. EDAX spectrum revealed the X-ray mediated electronic transitions corresponding to carbon $(\mathrm{C})$, nitrogen $(\mathrm{N})$ and oxygen $(\mathrm{O})$ along with the abundant peak for gold $(\mathrm{Au})$ (Figure 4a). The appearance of these peaks can be attributed to amino acid and this is possible only when amino acid shields the nanoparticle surface.

To quantify the amount of D-serine that was encapped in the particle, we performed an indirect assay, wherein we quantified the amount of unreacted amino acid after complete removal of nanoparticle from the post-reaction mixture by ninhydrin-based assay. The standard curve generated with the known concentration of amino acid was used to calculate the concentration of unreacted amino acid (Supplementary Figure S1). The amino acid concentration in the nanoparticle free reaction supernatant was estimated to be $60 \pm 4.0 \mathrm{mM}$. Since $100 \mathrm{mM}$ amino acid was used as starting material, this result indicated that $40 \%$ of the amino acid has been utilized for the encapment of GNP. It also further supports the view that D-serine shields GNP surface.

Stability of a nanoparticle is an important characteristic for its application and for this purpose we assessed the electro kinetic property developed at the electric double layer surrounding the particle by measuring the zeta potential, which is a known indicator for nanoparticle stability. Thus the particle generated by reducing with $100 \mathrm{mM}$ D-serine was subjected to zeta potential analysis and the zeta potential was found to be $-20.49 \mathrm{mV}$ (Figure $4 \mathrm{~b}$ ). This result indicates that this nanoparticle is stable.

To further determine the stability of this particle with respect to its salt tolerance, we exposed the nanoparticle to different concentrations of $\mathrm{NaCl}$ and determine the minimum concentration needed for the onset of aggregation. The minimum salt concentration at which the particle starts aggregating is known as critical flocculation concentration. We observed a red shift of absorption maxima at $540 \mathrm{~nm}$, characteristic of GNP, with a concomitant reduction in the optical density with the increasing concentration of sodium chloride (Figure 4c). Finally, at $1 \mathrm{M}$ of sodium chloride the solution turned bluish-purple and showed a shifted absorption maxima at around $650 \mathrm{~nm}$ which is characteristic of aggregation (Figure 4c). Taken together, these results again indicate that the GNP-conjugated with the amino acid is stable even at high salt concentration.

\section{Anti-mycobacterial activity of D-serine is enhanced when delivered with GNP}

D-serine is known to have anti-mycobacterial activity and thus wanted to determine if the D-serine protected GNP exhibits similar activity. The anti-mycobacterial activity of the $\mathrm{D}$-serine coated nanoparticle was assessed against $M$. smegmatis as described in Materials and Methods. D-serine alone was able to inhibit the growth of $M$. smegmatis at a minimum concentration of $24 \mathrm{mM}$ (Table 1, Supplementary Figure S2). Whereas, GNP preparation that contains $1 \mathrm{mM}$ equivalent of $\mathrm{D}$-serine was able to inhibit the growth of $M$. smegmatis (Table 1, Supplementary Figure S2). The approximate amount of D-serine that was coated on GNP was estimated by ninhydrin experiment as described in materials and methods. The result showed an enhanced ( 24 fold) anti-mycobacterial activity of GNP-conjugated D-serine compared to D-serine only.

\section{Discussion}

In this study we have described the synthesis of chiral GNP by direct reduction with both the enantiomeric forms of amino acid 
(a)

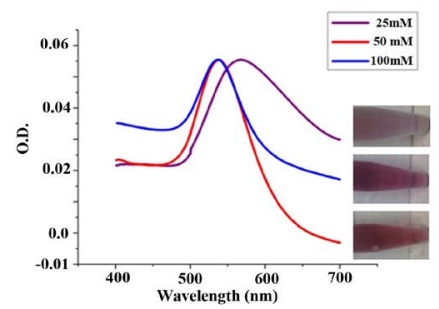

(c)

(b)

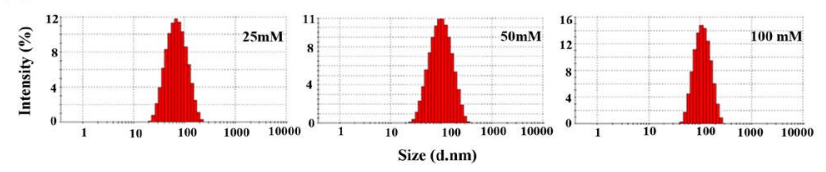

Figure 2: Physical characterization of GNPs synthesized using different concentrations of L-serine. (a) Colour and SPR of GNPs. Different concentrations of L-serine are indicated with different coloured lines: blue line indicates $100 \mathrm{mM}$, red line indicates $50 \mathrm{mM}$ and purple line indicates $25 \mathrm{mM}$. (b) Average hydrodynamic radius of GNPs. Average hydrodynamic radiuses of GNPs were determined by DLS (left panel is for $25 \mathrm{mM}$, middle panel is for $50 \mathrm{mM}$, and right panel is for $100 \mathrm{mM}$ ). (c) Transmission electron microscopic image of GNP synthesized using $100 \mathrm{mM}$ L-serine. Shape of a single particle is showed in inset.

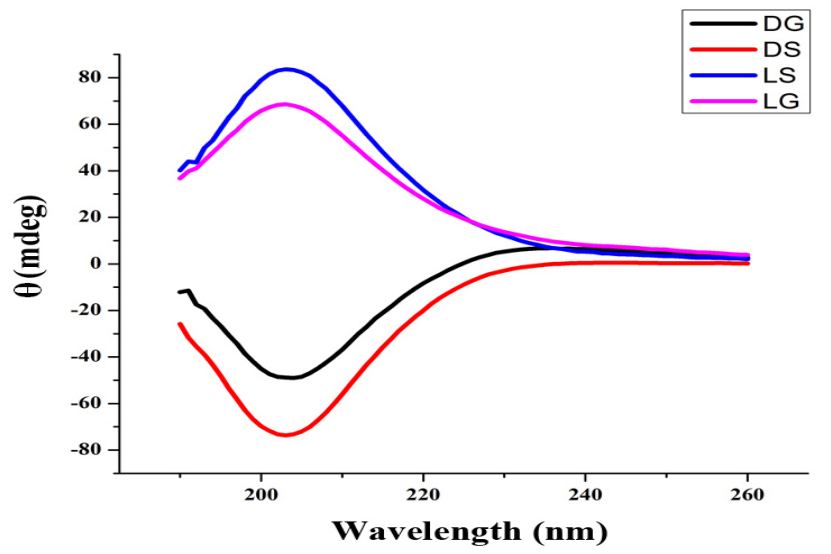

Figure 3: CD spectroscopy profile. CD spectra of both pure L-serine (LS) and D-serine (DS) and corresponding GNPs (LG and DG, respectively)

\begin{tabular}{|c|c|}
\hline Agent & MIC (mM) \\
\hline L-serine & N.D \\
\hline GNP with L-serine & N.D \\
\hline D-Serine & 24 \\
\hline GNP with D-serine & 1 \\
\hline
\end{tabular}

Table 1: GNP-conjugated D-serine exhibits enhanced anti-mycobacterial activity against $S$. smegmatis. N.D: Not detected even at quite high concentration.

serine. D-serine protected GNP showed enhanced activity against mycobacteria than D-serine alone suggesting a better delivery of the D-serine within the bacteria with the aid of GNP.

We opted for GNP due to its ease of preparation, controllable particle size and good solubility in wide range of buffer solution, surface modification and excellent biocompatibility. Towards the effort of replacing hazardous reducing agent by those that are biocompatible and green, in the present study we used amino acids; D- and L-serine. Although several scientists have used different amino acids for the reduction and protection of metal nanoparticles [8-12], this is the first report of direct reduction of tetrachloro auric acid by both the enantiomeric forms of serine. In this study we have also confirmed that the chirality is retained even after the GNP is being shielded with the amino acid. SEM-EDAX experiment clearly showed the presence of amino acid on the GNP surface. Probably, the chiral centre of the amino acid remained unaffected in the reduction process and thus this amino acid shielded particle showed stable chirality even after the reduction process. This kind of chiral nanoparticles may prove to be useful in application where enantioselectivity plays a major role. Moreover, though the reduction and shielding of metal nanoparticle with antibiotics and/or drugs and their subsequent activity enhancement is known [13-16], the capping of GNP with enantiomeric agent harbouring activity against bacteria can prove to be useful in future in the sphere of public health management. Towards this our result has already documented an increased activity of GNPcoated with $\mathrm{D}$-serine against $\mathrm{M}$. smegmatis, an avirulent laboratory strain of Mycobacterium origin.

Generally the encapment efficiency of any agent is assessed by tedious method like HPLC etc. In this study we have established a very simple ninhydrin-based method to calculate the shielding efficiency that works only when amino acids are used as reducing agent. This assay if not as accurate as chromatographic method, but still gives a fair estimation of amino acid associated with the reduced nanoparticle in a more faster and cost effective way. Therefore in this study we not only deciphered the nature of protection by techniques like SEM-EDAX, but also estimated the amount of amino acid still associated with the metal nanoparticle that helped to calculate the fold change in activity.

The activity of nanoparticles is highly dependent on its shape and size. Size of the particle is important for its transport and cellular permeability [32-34]. Nanoparticle size affects the uptake efficiency and kinetics, internalization mechanism and the subcellular distribution. For example, particle of less than $12 \mathrm{~nm}$ in size can cross the blood-brain barrier, whereas particle less than $30 \mathrm{~nm}$ in size can be endocytosed by cell [35]. Consistently size dependent variations in the uptake of GNPs in different cell lines have been reported with the maximum uptake in the range of 30 to $50 \mathrm{~nm}$ [34]. Similarly nano particle size also affects cytotoxicity after internalization. Normally smaller nanoparticles appear more toxic than the corresponding larger ones. Towards this, GNPs of $1.4 \mathrm{~nm}$ size was shown to be highly toxic and caused rapid cell death. In contrast, the larger GNP (size $15 \mathrm{~nm}$ ) displayed low toxicity [36]. In a similar study, Connor et al. showed that GNPs of 18 $\mathrm{nm}$ size were taken up easily by human leukemic cell without causing toxic effects [37]. These observations suggest that GNPs that are 15-18 $\mathrm{nm}$ or more in size are less toxic. Thus, nanoparticles generated in this study exhibiting an average size of $29.6 \pm 4.78 \mathrm{~nm}$ and $52.8 \pm 5.33 \mathrm{~nm}$ are well commensurate with the size of particle reported to cross the cellular barrier and having almost no toxicity. Moreover, it remains stable even at considerably high salt concentration $(\sim 800 \mathrm{mM})$ with respect to physiological salt concentration $(\sim 154 \mathrm{mM})$ and thus holds the promise to be used in biological milieu. Thus this nanoparticle also holds the promise to be used as delivery vehicle for different bioactive agent especially with special reference to enantioselective activity. In our study as it has effectively killed the Mycobacteria, we assume that it has at least crossed mycobacterial cell wall which is one of the most complexes in structure of its kind. Besides exhibiting the antimycobacterial property, D-serine is a recommended drug for some neurological disorders [38,39]. Therefore, an efficient and targeted delivery of such an important molecule holds great significance in the field of drug delivery. In reference to Mycobacteria higher order 
(a)

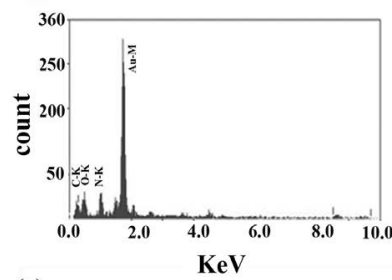

(c)
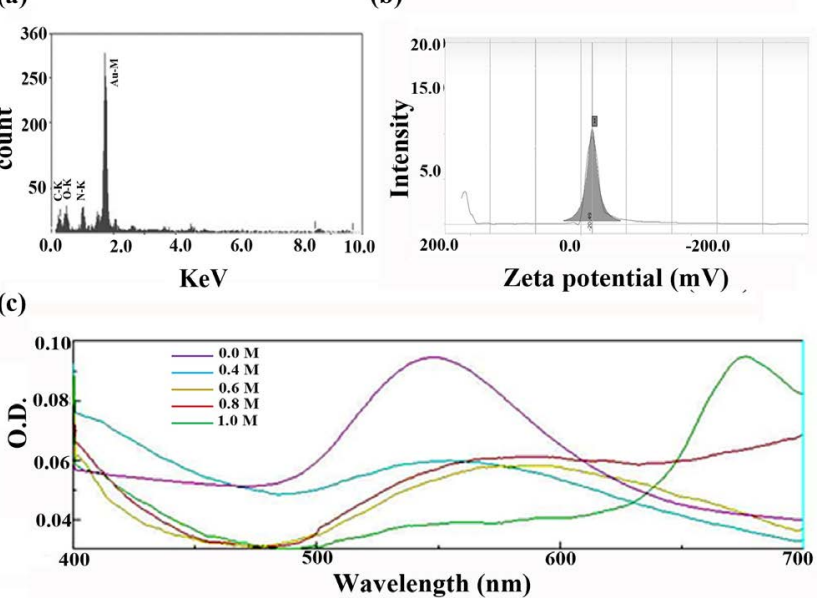

Figure 4: Characterization of the shielding of the GNP. (a) SEM-EDAX spectrum of the GNP synthesized by direct reduction with D-serine. (b) Zeta potential and other electrokinetic parameters of the GNP reduced by $100 \mathrm{mM}$ $\mathrm{D}$-serine (c) UV-Vis spectra of GNPs incubated with varying concentrations of $\mathrm{NaCl}$

antibiotics like $\mathrm{D}$-cycloserine that is derived from $\mathrm{D}$-serine itself can also expected to be delivered along with GNP. Similarly this method can be modified and extended to increase the efficacy other antimycobacterial compounds.

In conclusion, the current study describes the protocol of synthesis and protection of GNP with biocompatible reducing agent. In addition, the protecting agent retains its enantioselectivity and is stable enough in physiological condition to execute its anti-mycobacterial activity in a more efficient manner. Thus this study opens up a new but Simple Avenue that might ease the complexity of nanoparticle assisted antibacterial drug delivery in future.

\section{Acknowledgements}

Authors would like to acknowledge Dr. Aparna Laskar, IICB, Kolkata, India for helping in TEM experiment, Dr, Gautam Basu, Department of Biophysics, Bose Institute, Kolkata, India for providing the CD spectroscopy facility. We would also like to thank Prof. Anjan Dasgupta and Dr. Shibsekhar Roy for their valuable comments and suggestions. KM was supported by an institutional fellowship. This work has partly been supported by a grant from Centre for Research in Nanoscience and Nanotechnology, University of Calcutta.

\section{References}

1. Sperling RA, Rivera Gil P, Zhang F, Zanella M, Parak WJ (2008) Biological applications of gold nanoparticles. Chem Soc Rev 37: 1896-1908.

2. Kang YJ, Oh JW, Kim YR, Kim JS, Kim H (2010) Chiral gold nanoparticlebased electrochemical sensor for enantioselective recognition of 3,4-dihydroxyphenylalanine. Chem Commun (Camb) 46: 5665-5667.

3. Turkevich J, Steveson PC, Hillier J (1951) A study of the nucleation and growth processes in the synthesis of colloidal gold. Discuss Faraday Soc 11: 55-75.

4. Brust M, Walker M, Bethell D, Schiffrin, DJ, Whyman R (1994) Synthesis of thiol-derivatised gold nanoparticles in a two-phase liquid-liquid system. J Chem Soc Chem Commun 1994: 801-802.

5. Leff DV, Brandt L, Heath JR (1996) Synthesis and characterization of hydrophobic, organically-soluble gold nanocrystals functionalized with primary amines. Langmuir 12: 4723-4730.

6. Iwamoto M, Kuroda K, Zaporojtchenko V, Hayashi S, Faupel F (2003) Production of gold nanoparticles-polymer composite by quite simple method. Eur Phys JD 24: 365-367.

7. Potara M, Maniu D, Astilean S (2009) The synthesis of biocompatible and SERS-active gold nanoparticles using chitosan. Nanotechnology 20: 315602.
8. Polavarapu L, Xu QH (2008) A single-step synthesis of gold nanochains using an amino acid as a capping agent and characterization of their optical properties. Nanotechnology 19: 075601.

9. Wangoo N, Bhasin KK, Mehta SK, Suri CR (2008) Synthesis and capping of water-dispersed gold nanoparticles by an amino acid: bioconjugation and binding studies. J Colloid Interface Sci 323: 247-254.

10. Selvakannan PR, Mandal S, Phadtare S, Pasricha R, Sastry M (2003) Capping of gold nanoparticles by the amino acid lysine renders them water-dispersible. Langmuir 19: 3545-3549.

11. Selvakannan P, Mandal S, Phadtare S, Gole A, Pasricha R, et al. (2004) Water-dispersible tryptophan-protected gold nanoparticles prepared by the spontaneous reduction of aqueous chloroaurate ions by the amino acid. $J$ Colloid Interface Sci 269: 97-102.

12. Mandal S, Selvakannan PR, Phadtare S, Pasricha R, Sastry M (2002) Synthesis of a stable gold hydrosol by the reduction of chloroaurate ions by the amino acid, aspartic acid. J Chem Sci 114: 513-520.

13. Saha B, Bhattacharya J, Mukherjee A, Ghosh A K, Santra C (2007) In vitro structural and functional evaluation of gold nanoparticles conjugated antibiotics. Nanoscale Res Lett 2: 614-622.

14. Ahmad Z, Sharma S, Khuller GK (2005) Inhalable alginate nanoparticles as antitubercular drug carriers against experimental tuberculosis. Int $\mathrm{J}$ Antimicrob Agents 26: 298-303.

15. Pandey R, Sharma A, Zahoor A, Sharma S, Khuller GK, et al. (2003) Poly (DL-lactide-co-glycolide) nanoparticle-based inhalable sustained drug delivery system for experimental tuberculosis. J Antimicrob Chemother 52: 981-986.

16. Sung JC, Pulliam BL, Edwards DA (2007) Nanoparticles for drug delivery to the lungs. Trends Biotechnol 25: 563-570.

17. Bolla JM, Alibert-Franco S, Handzlik J, Chevalier J, Mahamoud A, et al. (2011) Strategies for bypassing the membrane barrier in multidrug resistant Gramnegative bacteria. FEBS Lett 585: 1682-1690.

18. Patra P, Mitra S, Debnatha N, Pramanikb P, Goswamia A (2014) Ciprofloxacin conjugated zinc oxide nanoparticle: A camouflage towards multidrug resistant bacteria. Bull Mater Sci 37: 199-206.

19. Lara H, Ayala-Núñez N, Ixtepan TL, Rodríguez PC (2010) Bactericidal effect of silver nanoparticles against multidrug-resistant bacteria. World J Microbiol Biotechnol 26: 615-621.

20. Maher TJ, Johnson DA (1991) Review of chirality and its importance in pharmacology. Drug Dev Res 24: 149-156.

21. Sawai K, Tatumi R, Nakahodo T, Fujihara H (2008) Asymmetric suzukimiyaura coupling reactions catalyzed by chiral palladium nanoparticles at room temperature. Angew Chem Int Ed Engl 47: 6917-6919.

22. Shukla N, Bartel MA, Gellman AJ (2010) Enantioselective separation on chiral Au nanoparticles. J Am Chem Soc 132: 8575-8580.

23. Yang L, Chen C, Liu X, Shi J, Wang G, et al. (2010) Use of cyclodextrin-modified gold nanoparticles for enantioseparations of drugs and amino acids based on pseudostationary phase-capillary electrochromatography. Electrophoresis 31 : 1697-1705.

24. Qi H, Hegmann T (2011) Liquid crystal-gold nanoparticle composites. Liquid Cryst 20: 102-114.

25. Suedee R, Jantarat C, Lindner W, Viernstein H, Songkro S, et al. (2010) Development of a $\mathrm{pH}$-responsive drug delivery system for enantioselectivecontrolled delivery of racemic drugs. J Control Release 142: 122-131.

26. Preiss LC, Werber L, Fischer V, Hanif S, Landfester K, et al. (2015) Aminoacid-based chiral nanoparticles for enantioselective crystallization. Adv Mater 27: 2728-2732.

27. Contino A, Maccarrone G, Zimbone M, Musumeci P, Calcagno L, et al. (2015) Fine tuning the $\mathrm{pH}$ triggers the enantiorecognition of underivatized amino acids by silver nanoparticles: a novel approach based on the focused use of solution equilibria. J Colloid Interface Sci 443: 30-35

28. Dolamic I, Varnholt B, Bürgi T (2015) Chirality transfer from gold nanocluster to adsorbate evidenced by vibrational circular dichroism. Nat Commun 6: 7117.

29. Kumar VB, Mastai Y, Porat Z, Gedanken A (2015) Chiral imprinting in molten gallium. New J Chem 39: 2690-2696.

30. Yabu K, Huempfner HR (1974) Inhibition of growth of Mycobacterium smegmatis 
Citation: Mukherjee K, Sil AK (2015) Synthesis of Chiral Gold Nanoparticle by Direct Reduction with L and D-Serine and Enhanced Anti-Mycobacterial Activity by D-Serine Protected Gold Nanoparticle. Mod Chem appl 3: 165. doi:10.4172/2329-6798.1000165

Page 6 of 6

and of cell wall synthesis by D-serine. Antimicrob Agents Chemother 6: 1-10.

31. Huang X, El-Sayed (2010) Gold nanoparticles: Optical properties and implementation $\mathrm{s}$ in cancer diagnosis and photothermal therapy. J Adv Res 1: $13-28$.

32. Alkilany AM, Murphy CJ (2010) Toxicity and cellular uptake of gold nanoparticles: what we have learned so far? J Nanopart Res 12: 2313-2333.

33. Chithrani BD, Ghazani AA, Chan WC (2006) Determining the size and shape dependence of gold nanoparticle uptake into mammalian cells. Nano Lett 6: 662-668.

34. Shang L, Nienhaus K, Nienhaus GU (2014) Engineered nanoparticles interacting with cells: size matters. J Nanobiotechnology 12: 5.
35. Conner SD, Schmid SL (2003) Regulated portals of entry into the cell. Nature 422: 37-44.

36. Pan Y, Neuss S, Leifert A, Fischler M, Wen F, et al. (2007) Size-dependent cytotoxicity of gold nanoparticles. Small 3: 1941-1949.

37. Connor EE, Mwamuka J, Gole A, Murphy CJ, Wyatt MD (2005) Gold nanoparticles are taken up by human cells but do not cause acute cytotoxicity. Small 1: 325-327.

38. Tuominen HJ, Tiihonen J, Wahlbeck K (2005) Glutamatergic drugs for schizophrenia: a systematic review and meta-analysis. Schizophr Res 72: 225234

39. Sehgal SA, Khattak NA, Mir A (2013) Structural, phylogenetic and docking studies of D-amino acid oxidase activator (DAOA), a candidate schizophrenia gene. Theor Biol Med Model 10: 3 . 